\title{
Lifting degeneracy in nematic liquid crystal viscosities with a single optical measurement
}

\author{
T.P. Bennett ${ }^{\mathrm{a}}$, M. B. Proctor ${ }^{\mathrm{b}, 1}$, M. Kaczmarek ${ }^{\mathrm{b}}$, G. D’ Alessandro $^{\mathrm{a}}$ \\ ${ }^{a}$ Mathematical Sciences, University of Southampton, Southampton, England, UK \\ ${ }^{b}$ Physics and Astronomy, University of Southampton, Southampton, England, UK
}

\begin{abstract}
The viscosity of complex, anisotropic fluids, such as liquid crystals or their colloidal suspensions, is characterized by a number of coefficients. Methods to measure them are, typically, sensitive only to their particular combinations, hence unable to determine them individually. Using an Ericksen-Leslie model and propagation of light through aligned layers of such materials, we show theoretically and verify experimentally how this degeneracy can be lifted by exploiting both the amplitude and frequency of the voltage applied to the cell as control parameters.
\end{abstract}

Keywords: Liquid crystals; Viscosity measurement; Viscosity of liquid crystals; Optics of liquid crystals; Ericksen-Leslie; Viscosity of complex fluids

\section{Introduction}

Viscosity and visco-elastic coefficients play an important role in the dynamics of soft matter either in the form of pure complex fluids or of suspensions of micron or nanometer size objects. For example, when nanoscale objects, such as carbon nanotubes [1] or inorganic nanoparticles [2] were dispersed in liquid crystals, the host viscosity as well as elastic constants were modified. In another study, Faucheux et al [3] used an asymmetric potential in optical tweezers to realize a thermal ratchet whose time scales depend on the particle diffusion coefficient. Hough and Ou-Yang |4| also experimented with optical tweezers to trap two particles in a viscous fluid and study the fluid-induced correlations between their periodic motion. Viscosity is also very important in biological suspensions: for example, Chengala and co-workers explored the role of viscous shear in the flow of alga in microfluidic channels [5].

In many of these examples light was used to analyze the system response to induced motion. Here we adopt a similar principle to study the viscosity of an optically active system. We set the system in motion using a periodic force and measure its response by detecting the effect on the intensity of a light beam propagating through it. The system we focus on as a demonstration of principle is a nematic liquid crystal (LC) cell forced by an oscillating AC electric field. However, this technique could, in principle, be applied to other systems. For example, it could be particularly useful for characterizing colloidal suspensions of inorganic, plasmonic, ferroelectric or ferromagnetic nanoparticles.

The interest in LCs stems from their ubiquity in optoelectronic applications, where their birefringence and reorientation properties make them materials of choice for electrically tunable optical devices. Moreover, the speed at which LC molecules

\footnotetext{
${ }^{1}$ Current address: NKT Photonics, Unit 20 Compass Point, Ensign Way, Southampton SO31 4RA
}

respond to a field is a critical property when considering device designs or new LC materials and it is, therefore, important to develop simple and quick methods to measure their viscosity and, hence, their response time.

In fact, the time response of an isotropic liquid to an external stimulus is inversely proportional to its viscosity. LCs are anisotropic liquids and their flow behavior is described by more than one viscosity coefficient, as is the case for the EricksonLeslie-Parodi [6-8] theory for the director flow and alignment, which uses the five Leslie viscosity coefficients, $\alpha_{1-5}$, though not all of them are equally critical in determining the switching dynamics of an LC device. For example, to first approximation the switch-off time of a planar LC cell of thickness $d$ is given by $|7|$

$$
\tau_{\mathrm{off}}=\frac{d^{2} \gamma_{1}}{K_{1} \pi^{2}},
$$

where $K_{1}$ is the splay elastic constant of the LC and $\gamma_{1}$ is its rotational viscosity, which can be written in terms of the Leslie viscosities as $\gamma_{1}=\alpha_{3}-\alpha_{2}$. Hence, determining the response time relies on accurately measuring several Leslie coefficients and, in particular, $\gamma_{1}$.

As the complexity of new LCs, as well as different electrode geometries, increases to achieve faster and stronger reorientation, the contribution and the values of different Leslie viscosities can play an important role. A typical example is the "kickback effect" in a splay cell [6]: when a large field is applied, the nematic in the center of the cell aligns. However, at the edges of the cell, a thin layer exists in which the director is rotated through $\pi / 2$ radians with respect to the alignment at the center in order to obey the boundary conditions. When the external field is switched off, the director begins to relax at the sides of the cell, where the distortion is large. This reorientation induces shear fluid flow, transferring angular momentum towards the center of the cell. This results in the counter-intuitive situation where the distortion at the center of the cell initially in- 
creases before relaxing. This kick-back effect, and the resulting reorientation angle, can be particularly pronounced if a large electric or magnetic field is applied and then turned off. This angle can be analytically related to the Leslie viscosity coefficients $\alpha_{2,4,5}[6,9]$.

There are various approaches to determine some combination of the Leslie viscosity coefficients, including mechanical methods $[10,11]$ such as measuring the damping of a submerged oscillating plate under different director fields. Optical methods, used either in splay geometry [12] or in twisted cells [13], rely on measuring the transient optical response of a nematic under the application of a step-wise voltage. There are a number of advantages and drawbacks for each technique: direct mechanical methods tend to give the most accurate measurements of viscosity coefficients, but rely on bespoke equipment; measuring the transient optical response requires a smaller volume of LC |11| with respect to mechanical methods, but places undue weight on the initial director configuration. Scattering experiments $[11,14 \mid$, provide accurate measurements of $\gamma_{1}$, but requires an uncommon optical set-up. In another approach, the oscillatory response of a LC subjected to a static magnetic field and a sinusoidal electric field has been studied using NMR measurements [15]. Interestingly, in this work the oscillations of the director could be modeled neglecting fluid motion.

In this paper we describe a new, efficient and accurate optical method to determine the LC dynamical properties using light as a measuring tool and the frequency and amplitude of an applied AC field as the control parameters. It is known in the literature that optical measurements cannot easily separate the effects of different viscosity parameters and, hence, measure them. Kelly et al [13| use cross-polarized intensity (CPI) measurements of switch-on and switch-off dynamics in a twisted cell to measure $\gamma_{1}$ and $\alpha_{4}+\alpha_{5}$. They use low and large amplitude switching to measure $\gamma_{1}$ and $\alpha_{4}+\alpha_{5}$, respectively. This paper builds on earlier work by Cossalter et al [16, 17] which suggested that all viscosities could be measured using switchon and switch-off dynamics. Kelly et al doubt this statement, but do not labor this point further. Grinfeld et al [9] show very elegantly that a two-mode linearised solution of the EricksenLeslie equations in a planar cell is able to describe quite accurately the kick-back dynamics. The authors indicate that it could be possible to use this solution to obtain physical parameters of the LC, but do not dwell further on this point except to note that the quantity that is normally measured is the CPI rather than the LC deflection and this may affect the fitting procedure. This is indeed a key point, as we illustrate in this paper.

In the next section we introduce an Ericksen-Leslie model of the director dynamics in a planar LC cell and its effect on the CPI of a light beam propagating through the cell. In section 3 we explain how CPI measurements suffer from degeneracy in the viscosity coefficients and show how this can be lifted using the frequency and amplitude of the voltage applied to the cell as control parameters. This is, in a narrow sense, the frequency domain equivalent of the time-domain technique of Kelly et al [13], but the detailed analysis in this section allows us to put it on firmer grounds, while the ease of control

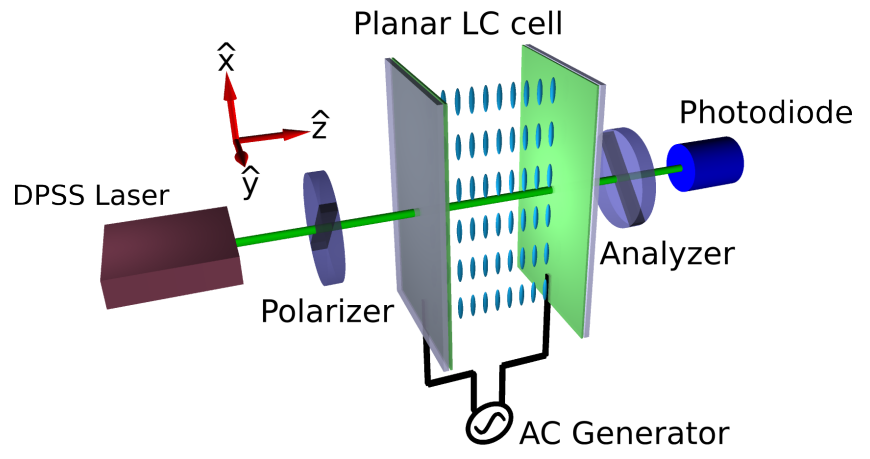

Figure 1: Schematic representation of the experimental set-up to measure the $\mathrm{CPI}$ as a function of the amplitude and frequency of the voltage applied across a planar LC cell. In this setup the polariser and analyzer are perpendicular to each other and the laser light is emitted by a diode pumped solid state (DPSS) laser.

of the frequency of the driving voltage gives us great flexibility in choosing optimal measurement regimes. We use this new procedure to measure the viscosities of some standard LCs: we describe the experimental set up in section 4 and the fitting procedure in section 5 . The paper is closed by a discussion of the advantages and disadvantages of the method and its possible extensions.

\section{Flow in an AC-driven planar cell}

Optical methods to determine elastic constants, pretilt, birefringence and cell thickness are based on CPI measurements (see figure 1 for a typical experimental setup). Normally, in these experiments the frequency of the applied voltage has a high value $(1-10 \mathrm{kHz})$ so that the LC responds only to the rms voltage amplitude and the CPI is constant in time. We extend this method by allowing the frequency to have sufficiently low values so that the $\mathrm{LC}$ is able to follow the driving voltage: the director oscillates about an average deflection, determined by the amplitude of the applied field, and modulates the phase lag of the light and, hence, the CPI. Its standard deviation is a good measure of the modulation amplitude. The director oscillations are coupled to the fluid flow within the cell and, as a result, optical measurements are sensitive to multiple viscosity coefficients.

To put this observation on a quantitative basis we use the Ericksen-Leslie theory to write a model for the LC and, hence, the CPI dynamics. The director field $\hat{\boldsymbol{n}}$ is identified by the angle $\theta$ that it forms with the $x$-axis, $\hat{\boldsymbol{n}}=[\cos (\theta(z)), 0, \sin (\theta(z))]$ where $x$ and $z$ are defined in figure 1 . In this configuration the fluid velocity of the nematic is given by $\boldsymbol{v}=[v, 0,0]$. The governing equations take the form of two non-linear coupled partial differential equations that describe the director and fluid dynamics [7|. The governing equation for the director describes a balance between electrostatic torque and the elastic restoring force in the nematic and includes a term coupling the director rotation to the fluid flow. The fluid flow is due entirely to the reorientation of the director. In dimensional form, the governing 
equations are $|7|$ :

$$
\begin{aligned}
& {\left[K_{1} \cos ^{2}(\theta)+K_{3} \sin ^{2}(\theta)\right] \frac{\partial^{2} \theta}{\partial z^{2}}+\frac{K_{3}-K_{1}}{2}\left(\frac{\partial \theta}{\partial z}\right)^{2} \sin (2 \theta)} \\
& +\frac{\epsilon_{a} \epsilon_{0}}{2}\left(\frac{\partial \phi(z, t ; \omega)}{\partial z}\right)^{2} \sin (2 \theta)-m(\theta) \frac{\partial v}{\partial z}=\gamma_{1} \frac{\partial \theta}{\partial t} \\
& \frac{\partial}{\partial z}\left[g(\theta) \frac{\partial v}{\partial z}+m(\theta) \frac{\partial \theta}{\partial t}\right]=0
\end{aligned}
$$

with

$$
m(\theta)=\frac{1}{2}\left[\left(\alpha_{3}-\alpha_{2}\right)+\left(\alpha_{3}+\alpha_{2}\right) \cos (2 \theta)\right]
$$

and

$$
\begin{aligned}
g(\theta)= & \frac{1}{2}\left(\alpha_{3}+\alpha_{2}\right) \cos (2 \theta)-\frac{\alpha_{1}}{8} \cos (4 \theta) \\
& +\frac{1}{2}\left(\alpha_{3}+\alpha_{4}+\alpha_{5}+\alpha_{1} / 4\right) .
\end{aligned}
$$

The electric potential is given by

$$
\phi(z, t ; \omega)=\int_{0}^{z} \frac{V(t ; \omega)}{1+\frac{\epsilon_{a}}{\epsilon_{\perp}} \sin ^{2}(\theta)} d z^{\prime}\left(\int_{0}^{d} \frac{1}{1+\frac{\epsilon_{a}}{\epsilon_{\perp}} \sin ^{2}(\theta)} d z^{\prime \prime}\right)^{-1}
$$

In these equations $K_{1}$ and $K_{3}$ are the splay and bend elastic constants, $\epsilon_{0}$ is the vacuum permittivity and $\epsilon_{a}=\epsilon_{\|}-\epsilon_{\perp}$ the dielectric anisotropy of the nematic, with $\epsilon_{\|}$and $\epsilon_{\perp}$ the component of permittivity along and orthogonal to the director respectively. The rotational viscosity $\gamma_{1}$ is related to the Leslie viscosity coefficients $\alpha_{i}, i=1, \ldots, 5$ by $\gamma_{1}=\alpha_{3}-\alpha_{2}$. For most LCs $\alpha_{1}$ and $\alpha_{3}$ are small in comparison to the other viscosity coefficients. The boundary conditions on equation (1) are that the director forms a small angle with the cell sides, i.e. the orientation is approximately planar: $\theta(0) \simeq \theta(d)=\theta_{0}$, where $d$ is the cell thickness and $\theta_{0}$ is a small angle assumed to be approximately the same on the two cell sides. We assume no-slip boundary conditions for the velocity.

With a given solution for $\theta(z, t)$, the CPI, $I(z, t)$ is given by

$$
I(z, t)=\sin ^{2}\left(\frac{\pi}{\lambda} \int_{0}^{d}\left[n_{0}-n_{\mathrm{eff}}(\theta)\right] d z\right),
$$

where $n_{\text {eff }}$ is the effective refractive index seen by the component of polarization in the plane of the director,

$$
n_{\mathrm{eff}}(\theta)=\frac{n_{e} n_{0}}{\sqrt{n_{0}^{2} \cos ^{2}(\theta)+n_{e}^{2} \sin ^{2}(\theta(z))}} .
$$

Here $n_{o}$ and $n_{e}$ are the optical ordinary and extra-ordinary refractive indexes respectively.

Under the application of an alternating potential, the LC reorients with the time-averaged director determined by the amplitude of the voltage. In addition, the director undergoes oscillations about the mean deflection which induce fluid flow that agitates the director throughout the cell and redistributes angular momentum. The oscillations in the director lead to modulations in the phase shift across the cell detectable in the dynamics of the CPI trace. The standard deviation of the CPI gives a measure of the dynamic response of the nematic and in principle we could expect to use it to measure the LC viscosities. In the next section we show that the situation is not so straightforward.

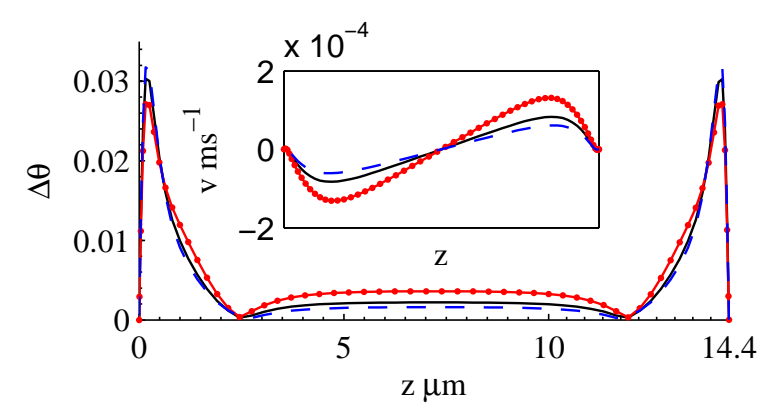

Figure 2: Amplitude of the director oscillations (outer plot) and velocity profile (insert) across a standard cell that correspond to the same value of the standard deviation of the CPI. The cell is driven by a $500 \mathrm{~Hz}$ sinusoidal voltage with a $20 \mathrm{~V}$ peak to peak amplitude. For the solid black line $\gamma_{1}=203.6 \mathrm{mPa}$ s and $\alpha_{4}+\alpha_{5}=431 \mathrm{mPa}$. The red dash-dot and the blue dash lines correspond to a change of $\gamma_{1}$ of $\pm 10 \%$ respectively. In both cases $\alpha_{4}+\alpha_{5}$ has been adjusted so that the standard deviations of the CPI was unchanged.

\section{Viscosity degeneracy}

In order to determine the limitation of CPI measurements, it is important to understand the role played by the major viscosities in equation (1) and (2): $\gamma_{1}$ sets the time scale on which the director responds to an applied field, while $\alpha_{4}+\alpha_{5}$ determines the strength of the coupling between the director and velocity field. Increasing the value of $\alpha_{4}+\alpha_{5}$ reduces the coupling between fluid flow and director dynamics. For a sinusoidal applied field, the measured signal is, in general, sensitive to both $\gamma_{1}$ and $\alpha_{4}+\alpha_{5}$ and these two parameters cannot be determined independently from standard CPI data. As an example, figure 2 shows the standard deviation of $\theta(z, t)$ and the velocity profile for different values of $\gamma_{1}$ and $\alpha_{4}+\alpha_{5}$, each of which produces the same value for the standard deviation of the CPI. The oscillations in $\theta$ shown in figure 2 towards the edges of the cell are driven directly by the alternating potential: here the LC alignment is not saturated by the strength of the electric field and the time variations of the latter can induce fluctuations in the former. These, in turn, induce the fluid flow responsible for driving the oscillations at the center of the cell. Decreasing the value of $\gamma_{1}$ primarily increases the oscillations at the edge of the cell as the nematic is more able to follow the alternating potential, while $\alpha_{4}+\alpha_{5}$ governs the amplitude of the oscillations at the center of the cell. Degeneracy in the measurement of $\gamma_{1}$ and $\alpha_{4}+\alpha_{5}$ is present because the optical measurements are insensitive to the region the oscillations are taking place in, and, as a result, it is possible to compensate for a change in $\gamma_{1}$ by changing $\alpha_{4}+\alpha_{5}$.

However, it is possible to break this degeneracy by operating at low voltage. To measure $\gamma_{1}$ we require that equation (1) is uncoupled from the flow velocity of the nematic, as in this way only $\gamma_{1}$ enters the governing equation for $\theta$. This can be achieved if the velocity gradient is small. From equation (2) we see that it is driven by the source term $m(\theta) \frac{\partial \theta}{\partial t}$. Examining equation (3) and using the assumption that $\alpha_{3}$ is small [18], a value of $\theta \approx 0$ will cause $m(\theta)$, and hence the source term in equation (2), to vanish.

As a result, oscillations in the director close to the $\theta=0$ 
state are sensitive only to $\gamma_{1}$ and their amplitude can be used to estimate this parameter. In order to have a detectable signal we also use a low frequency so that the amplitude of the director oscillations are relatively large, while the dynamics is sufficiently slow that no detectable flow is induced. Once $\gamma_{1}$ is known, $\alpha_{4}+\alpha_{5}$ can be estimated by the CPI oscillation amplitude at high voltage. In this case, in order to have a relatively simple CPI signal, consisting of sinusoidal oscillations around an average, it is convenient to use a higher frequency (of the order of $100 \mathrm{~Hz}$ ).

\section{Experimental setup}

To verify the validity of the method outlined in section 3 we have experimentally measured the CPI at different frequencies. Standard LC cells were prepared using ITO glass substrates, coated with rubbed polyimide (PI). They were filled with one of the three LCs selected for this investigation. The choice of LCs (E7, TL205, ZLI4792) was, first, driven by the availability in the literature of their physical, electrical and optical parameters, so that our results could be compared and verified. Indeed, E7 is one of the most comprehensively characterized nematic material. Secondly, TL205 and ZLI4792 represent a class of low ionic LCs [19] and proved promising as hosts for colloidal suspensions of $\mathrm{BaTiO}_{3}$ and $\mathrm{ZnS}$ nanoparticles, respectively $[2,20 \mid$. Thirdly, to test and validate our method, materials with different viscosities were needed and the three liquid crystals selected have different rotational viscosity parameters (see Table 1). Finally, we required them to be stable in the nematic phase at room temperature. The nematic to isotropic phase transition temperatures are $61{ }^{\circ} \mathrm{C}, 87.4{ }^{\circ} \mathrm{C}$ and $92{ }^{\circ} \mathrm{C}$, for E7, TL205 and ZLI4792, respectively. Furthermore, in order to test the method using a range of cell thicknesses, different sized spacer beads were used.

The cells were characterized using incident laser light at $532 \mathrm{~nm}$, polarized at an angle of $45^{\circ}$ with respect to the PI rubbing direction (see figure 1 ). The beam diameter was approximately $1 \mathrm{~mm}$ and the beam power $60 \mathrm{~mW}$. The phase difference between the two orthogonal components of this beam after passing through the cell was measured by adding an analyzer polariser, with transmission axis set orthogonal to the initial polarization, and observing the resulting changes in intensity using a photodiode.

The experiment had three aims: to determine the static parameters of the LC, to determine $\gamma_{1}$ and to determine $\alpha_{4}+\alpha_{5}$. The first step was to ramp the amplitude of a $10 \mathrm{kHz}$ voltage from 0-20 V peak to peak, and measure the average CPI at each voltage; the resulting data were used to fit values for $K_{1,3}$, the cell thickness, and pretilt. The fitting procedure for all of the experimental data is described in more detail below.

To measure $\gamma_{1}$ we used an applied potential of the form $V(t)=V_{a} \cos \left(2 \pi f_{H} t\right)\left[1+V_{b} \cos \left(2 \pi f_{l} t\right)\right]$, where $f_{H} \gg f_{l}$. This modulated signal allows an independent tuning of the mean deflection and of the oscillations of $\theta$. The high frequency carrier signal ensures that ion movement is negligible. The parameter $V_{a}$ sets the mean deflection of $\theta$ and $V_{b}$ provides control over the oscillations of $\theta$. Values of $V_{a, b}$ that result in a large variation of the CPI while inducing only a small variation of $\theta$ were chosen. Typically the voltage would vary by approximately $5-10 \%$ about a point close to the Frederiks transition. The frequency $f_{H}$ was set to $10 \mathrm{kHz}$, while the frequency $f_{l}$ was ramped in steps of $0.5 \mathrm{~Hz}$ from $0.1 \mathrm{~Hz}$ to $9.6 \mathrm{~Hz}$. $\gamma_{1}$ was then used to fit to the data.

The measurement of $\alpha_{4}+\alpha_{5}$ was performed by ramping the amplitude of a $500 \mathrm{~Hz}$ signal from $0 \mathrm{~V}$ to $20 \mathrm{~V}$ peak to peak in steps of $0.02 \mathrm{~V}$ (E7), $0.04 \mathrm{~V}$ (ZLI4792) and $0.05 \mathrm{~V}$ (TL205), waiting $5 \mathrm{~s}$ for the transient to elapse and measuring the CPI at each voltage value for $0.02 \mathrm{~s}$. The standard deviation of the signal at each point was extracted, and the resulting data were fitted by varying $\alpha_{4}+\alpha_{5}$.

\section{Fitting procedure and validation}

In order to compute the CPI, the governing equations (1) and (2) were solved in Matlab using the method detailed by Mottram et al [21] to reduce the coupled governing equations (1) and (2) to a single equation for $\theta$ containing a non local source term representing the flow. A spectral collocation method was used to discretize space [22], and an implicit variable step algorithm (Matlab routine ode15s) to integrate in time.

To compute the CPI the integral in equation (6) was computed using a Clencurt quadrature [22]. Its average and standard deviation were extracted to compare it with the experimental data.

Fitting of the experimental data was done in two steps. First, the average of the CPI taken at $500 \mathrm{~Hz}$ and $10 \mathrm{kHz}$ were fitted giving values for the cell size, elastic constants and pretilt. These values were then used when fitting the viscosities to the standard deviation of the oscillations of the CPI measured in the two regimes. The cell thickness $d$ determines the maximum polarization phase shift in propagation. The pretilt $\theta_{0}$ regulates how sharp the Frederiks transition is and, in conjunction with $d$, determines the zero voltage intensity. The splay elastic constant $K_{1}$ locates the Frederiks transition and controls the low voltage portion of the trace. The bend elastic constant $K_{3}$ only affects the high voltage behavior.

Fitting was done using a least square algorithm (Matlab routine lsqrcurvefit) as follows: first the cell thickness, pretilt and splay elastic constant were fitted to the low voltage portion of the trace (up to just above the Frederiks transition). With the values of the cell thickness and pretilt fixed the entire data trace was fitted using the two elastic constants as fitting parameters. The fitted values of thickness, pretilt and elastic constants determined from average of the $500 \mathrm{~Hz}$ and $10 \mathrm{kHz}$ data were used for subsequent fits to the standard deviation of the $500 \mathrm{~Hz}$ (amplitude modulated) data.

Once thickness, pretilt and elastic constants were known, the viscosities were obtained by a least square fit minimization. The fit was performed using the Matlab constrained optimization function fmincon and the active-set and sqp algorithms. Where available, we have used literature values of the viscosities as starting point for the minimization process (see table 1). Otherwise, reasonable guesses were used. To ensure convergence, we have used an iterative fitting procedure. 


\begin{tabular}{|c|c|c|c|c|c|c|}
\hline & $d$ & $\theta_{0}$ & $K_{1}$ & $K_{3}$ & $\gamma_{1}$ & $\alpha_{4}+\alpha_{5}$ \\
\hline E7 [12] & $14.4 \mu \mathrm{m}$ & $0.5^{\circ}$ & $10.9 \mathrm{pN}$ & $18.0 \mathrm{pN}$ & $\begin{array}{c}203.6 \mathrm{mPa} \mathrm{s} \\
(280.8 \mathrm{mPa} \mathrm{s})\end{array}$ & $\begin{array}{c}431 \mathrm{mPa} \mathrm{s} \\
(316.8 \mathrm{mPa} \mathrm{s})\end{array}$ \\
\hline TL205 |19| & $15.0 \mu \mathrm{m}$ & $1.22^{\circ}$ & $16.5 \mathrm{pN}$ & $20.3 \mathrm{pN}$ & $\begin{array}{l}303.0 \mathrm{mPa} \mathrm{s} \\
(367 \mathrm{mPa} \mathrm{s})\end{array}$ & $403.0 \mathrm{mPa} \mathrm{s}$ \\
\hline ZLI4792 |13| & $11.7 \mu \mathrm{m}$ & $1.78^{\circ}$ & $14.8 \mathrm{pN}$ & $19.8 \mathrm{pN}$ & $\begin{array}{l}109.6 \mathrm{mPa} \mathrm{s} \\
(95.0 \mathrm{mPa} \mathrm{s})\end{array}$ & $\begin{array}{c}133.4 \mathrm{mPa} \mathrm{s} \\
(115.0 \mathrm{mPa} \mathrm{s})\end{array}$ \\
\hline
\end{tabular}

Table 1: Cell and material parameters for E7, TL205 and ZLI4792 obtained from the fits shown in figures 35 The available literature values are reported in parenthesis below the corresponding fitted values. The literature parameters were estimated at $20.3^{\circ} \mathrm{C}, 20^{\circ} \mathrm{C}$ and "room temperature" respectively for the three $\mathrm{LCs}$. The uncertainty on the fitted data is likely to be of the order of tens of $\mathrm{mPa}$ s (see section 5 for more details); the error bars on the literature values are unknown.

The low voltage, low frequency measurements were fitted using only $\gamma_{1}$ as a fitting parameter and keeping $\alpha_{4}+\alpha_{5}$ fixed. With $\gamma_{1}$ found in this way, the high voltage data was used to determine $\alpha_{4}+\alpha_{5}$. With these updated values for $\gamma_{1}$ and $\alpha_{4}+\alpha_{5}$, the process was repeated to ensure convergence. The final fitted values of $\gamma_{1}$ and $\alpha_{4}+\alpha_{5}$ are shown in table 1

We have verified that the fitted values of $\gamma_{1}$, deduced from the low frequency measurements, are independent of $\alpha_{4}+\alpha_{5}$. To this end we have determined the minimization landscape in a neighborhood of the fitted values for $\gamma_{1}$ and $\alpha_{4}+\alpha_{5}$. The degeneracy between these two sets of parameters discussed in section 3 implies that the landscape has the shape of a valley steep in one direction, but fairly flat in the other. As discussed in section 3 , in general, increasing $\gamma_{1}$ while decreasing $\alpha_{4}+\alpha_{5}$ just moves the minimization point along the floor of the valley thus making it impossible to determine independently these two sets of parameters. However, in the case of the low frequency measurements that we have used to determine $\gamma_{1}$, the minimization valley is approximately parallel to the $\alpha_{4}+\alpha_{5}$ direction so that the fit is several orders of magnitude more sensitive to $\gamma_{1}$ than $\alpha_{4}+\alpha_{5}$, as expected (and desired).

The main objective of these measurements was to demonstrate the feasibility of our method and to check that the degeneracy between viscosity parameters could be lifted. The cell's temperature was assumed to be, approximately, the ambient temperature, which was $(22 \pm 2){ }^{\circ} \mathrm{C}$. In principle, for more accurate viscosity measurements, a dedicated, controlled stage could be used to ensure a constant cell temperature. In the literature, we found the viscosity temperature dependence only for E7. A Diogo-Martins model [23] of the temperature dependence of E7 fitted by $\mathrm{Wu}$ and $\mathrm{Wu}[12]$, suggests that in this temperature range $\gamma_{1}$ varies from approximately $280 \mathrm{mPa}$ s to approximately $180 \mathrm{mPa}$ s. Therefore, the difference between our and Wang et al's estimates, while large, is not unreasonable. We can assume that a similar range of uncertainty will also affect the other liquid crystals, even though possibly to a lower extent as they have a higher phase transition temperature.

The experimental data, along with the fits to Erickson-Leslie theory, are shown in figures 3.5 while the fitted parameters and comparison with the literature values are reported in Table 1 . These results show that the procedure outlined in this paper is

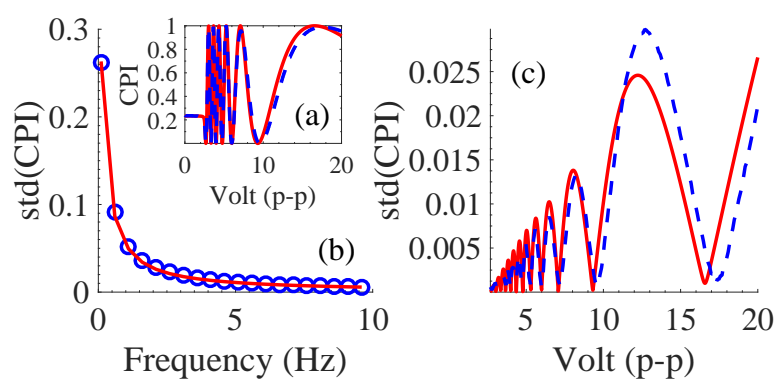

Figure 3: E7 measurements and fits - (a) measurement of the elastic constants, cell thickness and pretilt angle: average CPI as a function of peak to peak amplitude; (b) measurement of $\gamma_{1}$ : standard variation of the CPI as a function of frequency at a voltage close to the Fredericks transition; (c) measurement of $\alpha_{4}+\alpha_{5}$ : standard deviation of the CPI as a function of voltage at $500 \mathrm{~Hz}$. In all plots the blue markers or dashed lines refer to the experimental data, while the solid red lines are the theoretical fits.
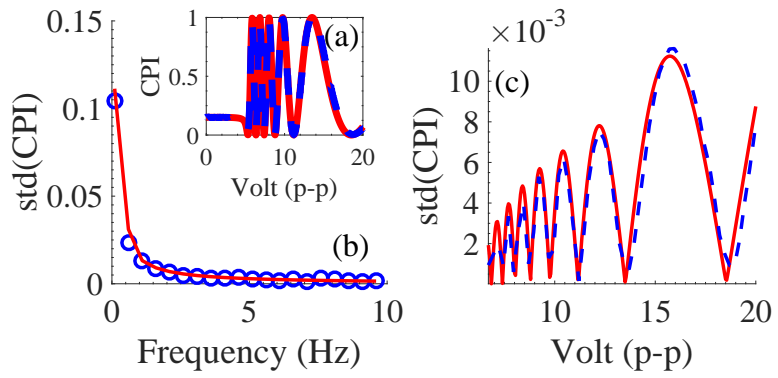

Figure 4: As in figure 3. except that the LC is TL205.
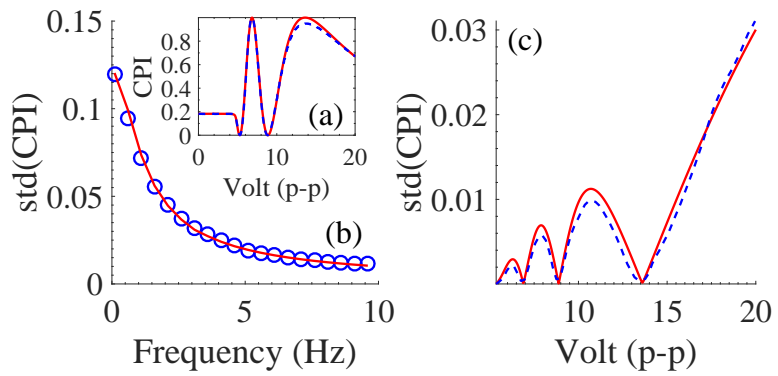

Figure 5: As in figure 3 except that the LC is ZLI4792. 
able to reliably measure Leslie viscosities that range between $100 \mathrm{mPa}$ s (ZLI4792) and $400 \mathrm{mPa}$ s (TL205 and E7). Moreover, these data indicate that the range of measurable viscosities is likely to be much larger. In general the theoretical fits agree well with the experimental results and the obtained viscosity values are physically reasonable and in good agreement with the literature. In fact, the only fit that requires some improvement is the estimate of $\alpha_{4}+\alpha_{5}$ for E7 in figure 3.(c). This fit suffers from the fact that there is slight misalignment in the fit to the average CPI (see figure 3.(a)). It is unclear what is the cause of this discrepancy, only present for E7 and high voltages. It is possible that fitting to the phase shift rather than the CPI may alleviate this problem, as the phase difference is a monotonic function of the applied voltage. An exploration of the optimal fitting procedure to minimize errors and increase automation is ongoing and will be reported in future work.

\section{Conclusions}

In this paper we have given a clear mathematical and physical explanation of the degeneracy between viscosities coefficients that affects CPI measurements reported in the literature $[13,16,17]$. In particular, we have shown that we should not just see it as an obstacle to accurate measurements, but also a key to understand the role that the different viscosities play in different regions of the cell. We have been able to lift it and, hence, obtain reliable estimates of $\gamma_{1}$ and $\alpha_{4}+\alpha_{5}$, by using the frequency of the applied voltage as a control parameter. This is a key advantage with respect to transient methods $[12,13]$ : by tuning the frequency we can probe the minimization landscape when fitting the liquid crystal parameters and ensure that the minimum obtained is isolated and, hence, the parameters fitted are uniquely determined.

The method we have proposed uses a single experimental set-up to measure several, important and fundamental static and dynamic parameters. The former are the cell thickness, pretilt angle, Frederiks transition, $K_{1}$ and $K_{3}$ elastic constants; the latter, the rotational viscosity $\gamma_{1}$ and the $\alpha_{4}+\alpha_{5}$ combination of Leslie viscosities. The static parameters are obtained from the average CPI trace at high frequency $(10 \mathrm{KHz})$. The dynamic parameters are derived from the small CPI oscillations at low or intermediate frequencies: $1-10 \mathrm{~Hz}$ for $\gamma_{1}$ and $100-500 \mathrm{~Hz}$ for $\alpha_{4}+\alpha_{5}$.

The method was verified by providing estimates of the values of $\gamma_{1}$ and $\alpha_{4}+\alpha_{5}$ for E7, TL205 and ZLI4792. In all cases we have shown that the degeneracy between viscosities can be lifted by using the frequency of the applied voltage as control parameter. Moreover, the values we have estimated are in agreement with those found in the literature, even though the cells used were not temperature stabilized. As an added bonus we have used the model to estimate also elastic constants, cell thickness, and pretilt.

In this article we have focused on nematic liquid crystals for reasons of availability and modeling simplicity. However, the method we propose is far more general and is likely to be of more use for non-standard fluids, like colloidal suspensions of inorganic nanoparticles in LCs. The key experimental advantages are (i) simplicity of the optical measurement, (ii) the use of only a small volume of suspension and, most importantly, (iii) the possibility of taking measurements in situ, without any need to extract the suspension from the cell. This allows, for example, to measure easily and repeatably the effect of aging on the static and dynamic properties of the suspension. Of course, the estimation of the parameters also requires a mathematical model of the alignment dynamics and of the fluid flow properties. Some extensions of the model are simple, e.g. to consider smectic liquid crystals for which an EricksenLeslie-Parodi theory exists $[7,24 \mid$. The extension to colloidal suspension is a much more interesting and open problem that is, ultimately, also more rewarding. One approach is to use a mean field theory similar to the models used to study the response to ferroelectric $[25]$ and ferromagnetic $[26,27]$ LC colloidal suspensions. In these theories the particles are treated as a small perturbation and the suspension equations are essentially those of a pure LC (with some additional coupling terms), but with parameters that can only be determined experimentally. Another approach is to start from a microscopic theory, e.g. using a Maier-Saupe approach [28] or homogenization methods [29], and obtain macroscopic theories whose parameters are fully determined by the microscopic interactions between particles and LC molecules. In both cases we can expect that using the frequency of the applied voltage as a control parameter will be a key requirement to help determine the model parameters and help discriminate between different theories.

\section{Acknowledgments}

The authors are grateful to Prof Tim Sluckin for his advice and careful reading of the manuscript. They would also like to thank the Royal Societys Paul Instrument Fund (PI150041) and the University of Southampton FSHMS Enterprise Fund for partial support of this research.

\section{References}

[1] N. Puech, C. Blanc, E. Grelet, C. Zamora-Ledezma, M. Maugey, C. Zakri, E. Anglaret, P. Poulin, Highly ordered carbon nanotube nematic liquid crystals, J.Phys. Chem. C 115 (8) (2011) 3272-3278. doi:10.1021/ jp1102077

[2] N. Podoliak, O. Buchnev, M. Herrington, E. Mavrona, M. Kaczmarek, A. G. Kanaras, E. Stratakis, J.-F. Blach, J.-F. Henninot, M. Warenghem, Elastic constants, viscosity and response time in nematic liquid crystals doped with ferroelectric nanoparticles, RSC Adv. 4 (2014) 46068-46074. doi : 10.1039/C4RA06248E

[3] L. P. Faucheux, L. S. Bourdieu, P. D. Kaplan, A. J. Libchaber, Optical thermal ratchet, Phys. Rev. Lett. 74 (1995) 1504-1507. doi:10.1103/ PhysRevLett.74.1504

[4] L. A. Hough, H. D. Ou-Yang, Correlated motions of two hydrodynamically coupled particles confined in separate quadratic potential wells, Phys. Rev. E 65 (2002) 021906. doi : 10.1103/PhysRevE.65.021906

[5] A. Chengala, M. Hondzo, J. Sheng, Microalga propels along vorticity direction in a shear flow, Phys. Rev. E 87 (2013) 052704. doi:10.1103/ PhysRevE.87.052704

[6] M. G. Clark, F. M. Leslie, A calculation of orientational relaxation in nematic liquid crystals, Proc. R. Soc. Lond. A 361 (1707) (1978) 463485. doi:10.1098/rspa.1978.0112

[7] I. W. Stewart, The static and dynamic continuum theory of liquid crystals: a mathematical introduction, Taylor \& Francis, 2004. 
[8] O. Parodi, Stress tensor for a nematic liquid crystal, J. Phys. France 31 (1970) 581-584. doi : 10.1051/jphys:01970003107058100

[9] M. Grinfeld, M. Langer, N. Mottram, Nematic viscosity estimation using director kickback dynamics, Liq. Cryst. 38 (8) (2011) 981-987. doi : 10.1080/02678292.2011.588969

[10] D. A. Dunmur, A. Fukuda, G. R. Luckhurst, Physical Properties of Liquid Crystals: Nematics, INSPEC, 2001

[11] V. V. Belyaev, Physical methods for measuring the viscosity coefficients of nematic liquid crystals, Physics - Uspekhi 44 (2001) 255-284. doi : 10.1070/PU2001v044n03ABEH000831

[12] H. Wang, T. X. Wu, S. Gauza, J. R. Wu, S.-T. Wu, A method to estimate the Leslie coefficients of liquid crystals based on MBBA data, Liq. Crys. 33 (1) (2006) 91-98. doi : 10.1080/02678290500446111

[13] J. Kelly, S. Jamal, M. Cui, Simulation of the dynamics of twisted nematic devices including flow, J. Appl. Phys. 86 (8) (1999) 4091-4095. doi : 10.1063/1.371333

[14] van der Meulen, J.P., Zijlstra, R.J.J., Physical properties of the nematic liquid crystal, apapa. optical determination of the viscoelastic and elastic ratios, J. Phys. France 45 (10) (1984) 1627-1642. doi:10.1051/ jphys : 0198400450100162700

[15] G. R. Luckhurst, T. Miyamoto, A. Sugimura, B. A. Timimi, H. Zimmermann, Electric-field driven director oscillations in a nematic liquid crystal: A NMR investigation J. Chem. Phys. 121 (4) (2004) 1928-1937. doi:10.1063/1.1764774

URL http://link.aip.org/link/?JCP/121/1928/1

[16] O. Cossalter, D. A. Mlynski, Determination of Leslie's viscosities with high accuracy directly from the electro-optic response of a LCD, Liq Cryst. 19 (4) (1995) 545-547. doi : 10.1080/02678299508032017

[17] O. Cossalter, B. Cramer, D. Mlynski, A new method of viscosity determination from the electro-optical response of a nematic LCD, J. Phys. II France 6 (12) (1996) 1663-1668. doi:10.1051/jp2:1996155

[18] P. Martinoty, S. Candau, Determination of viscosity coefficients of a nematic liquid crystal using a shear waves reflectance technique, Mol. Crys. and Liq. Crys. 14 (1971) 243-271.

[19] Merck KGaA, TL205 technical data sheet (2005).

[20] S. Acharya, S. Kundu, J. P. Hill, G. J. Richards, K. Ariga, Nanorod-Driven Orientational Control of Liquid Crystal for Polarization-Tailored ElectroOptic Devices. Adv. Mat. 21 (9) (2009) 989-993. doi : 10.1002/adma. 200802743 URL http://dx.doi.org/10.1002/adma. 200802743

[21] N. J. Mottram, J. T. Pinto, G. McKay, Decoupling of the EricksenLeslie equations, Liq. Cryst. 40 (6) (2013) 787-798. doi:10.1080/ 02678292.2013.783134

[22] L. N. Trefethen, Spectral Methods in Matlab, SIAM, Philadelphia, 2000.

[23] A. C. Diogo, A. F. Martins, Order parameter and temperature dependence of the hydrodynamic viscosities of nematic liquid crystals, J. Phys. France 43 (5) (1982) 779-786. doi : 10.1051/jphys: 01982004305077900

[24] I. W. Stewart, Dynamic theory for smectic a liquid crystals Continuum Mech. Thermodyn. 18 (2007) 343-360. URL http://link.springer.com/article/10.1007\% 2Fs00161-006-0035-4

[25] L. M. Lopatina, J. V. Selinger, Theory of Ferroelectric Nanoparticles in Nematic Liquid Crystals, Phys. Rev. Lett. 102 (2009) 197802. doi:10. 1103/PhysRevLett.102.197802

[26] S. V. Burylov, Y. L. Raikher, Macroscopic Properties of Ferronematics Caused by Orientational Interactions on the Particle Surfaces. II. Behavior of Real Ferronematics in External Fields, Mol. Cryst. Liq. Cryst. 258 (1995) 123-141. doi:10.1080/10587259508034553

[27] N. Podoliak, O. Buchnev, O. Buluy, G. D’Alessandro, M. Kaczmarek, Y. Reznikov, T. J. Sluckin, Macroscopic optical effects in low concentration ferronematics, Soft Matter 7 (10) (2011) 4742-4749. doi : 10.1039/C1SM05051F

[28] L. M. Lopatina, J. V. Selinger, Maier-Saupe-type theory of ferroelectric nanoparticles in nematic liquid crystals. Phys. Rev. E 84 (2011) 041703. doi:10.1103/PhysRevE.84.041703 URL http: //link.aps.org/doi/10.1103/PhysRevE. 84.041703

[29] T. P. Bennett, G. D'Alessandro, K. R. Daly, Multiscale models of colloidal dispersion of particles in nematic liquid crystals, Phys. Rev. E 90 (2014) 062505. doi:10.1103/PhysRevE.90.062505 